\title{
Sudden Cardiac Death in a Young Patient With Psoriasis
}

\author{
Gitesh U. Sawatkar, MD; Tarun Narang, MD; Valliappan Muthu, DM; Sunil Dogra, MD, FRCP; \\ Sanjeev Handa, MD, FRCP
}

\section{PRACTICE POINTS}

- Low-grade chronic inflammation in patients with psoriasis can lead to vascular inflammation, which can further lead to the development of major adverse cardiovascular events (CVEs) and arrhythmia.

- The need for a multidisciplinary approach and close monitoring of cardiovascular risk factors in patients with psoriasis to prevent a CVE is vital.

- Baseline electrocardiogram and biomarkers for cardiovascular disease also should be performed in young patients with severe or unstable psoriasis.

To the Editor:

The evolution in the understanding of psoriasis and psoriatic arthritis has unfolded many new facets of this immune-mediated inflammatory disease. Once considered to be just a cutaneous disease, psoriasis is not actually confined to skin but can involve almost any other system of the body. Cardiovascular morbidity and mortality are the major concerns in patients with psoriasis. We report the sudden death of a young man with severe psoriasis.

A 31-year-old man was admitted for severe psoriasis with pustular exacerbation (Figures $1 \mathrm{~A}$ and $1 \mathrm{~B}$ ). He had moderate to severe unstable disease during the last 8 years and was managed with oral methotrexate (0.3-0.5 $\mathrm{mg} / \mathrm{kg} / \mathrm{wk})$. He was not compliant with treatment, which led to multiple relapses. There was no personal or family history of risk factors for cardiovascular events (CVEs). At the time of present hospitalization, his vital parameters were normal. Physical examination revealed erythematous scaly plaques on more than $75 \%$ of the body surface area. Multiple pustules also were noted, often coalescing to form plaques (Figure 1C). Baseline investigations consisting of complete blood cell count, lipid profile, liver and renal functions, and chest radiography were within reference range. Baseline electrocardiogram (ECG) at admission was unremarkable (Figure 2A), except for sinus tachycardia. Low-voltage complexes in limb leads were appreciated as well as a corrected QT interval of 420 milliseconds (within reference range). Echocardiography was normal (visual ejection fraction of $60 \%$ ).

The patient was unable to tolerate methotrexate due to excessive nausea; he was started on oral acitretin $25 \mathrm{mg}$ once daily. There was no improvement in psoriasis over the following week, and he reported mild upper abdominal discomfort. He did not have any chest pain or dyspnea, and his pulse and blood pressure were normal. Serum electrolytes, liver function, lipid profile, and an ultrasound of the abdomen revealed no abnormalities. A repeat ECG showed no changes, and cardiac biomarkers were not elevated. Two days later, the patient collapsed while still in the hospital. A cardiac monitor and ECG showed ventricular tachycardia (VT)(Figure 2B); however, serum electrolytes, calcium, magnesium, and phosphorus levels were within reference range. Aggressive resuscitative measures

From the Postgraduate Institute of Medical Education and Research, Chandigarh, India. Drs. Sawatkar, Narang, Dogra, and Handa are from Department of Dermatology, Venereology and Leprology, and Dr. Muthu is from Department of Pulmonary Medicine.

The authors report no conflict of interest.

Correspondence: Tarun Narang, MD, Department of Dermatology, Venereology and Leprology, Postgraduate Institute of Medical Education and Research (PGIMER), Sector 12, Chandigarh 160012, India (narangtarun@yahoo.co.in).

doi:10.12788/cutis.0188 

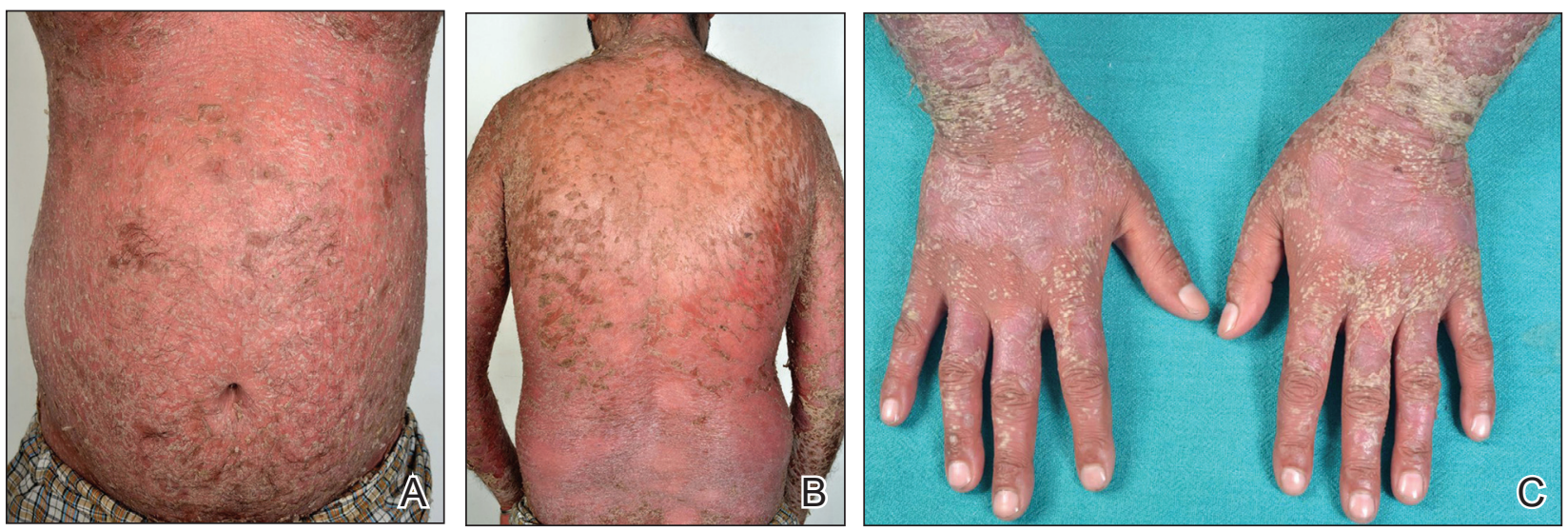

FIGURE 1. Severe plaque psoriasis. A, Erythematous, indurated, scaly plaques covering the chest and abdomen. B, Erythematous scaly plaques covering the back. C, Erythematous plaques with multiple pustules coalescing to form lakes of pus.
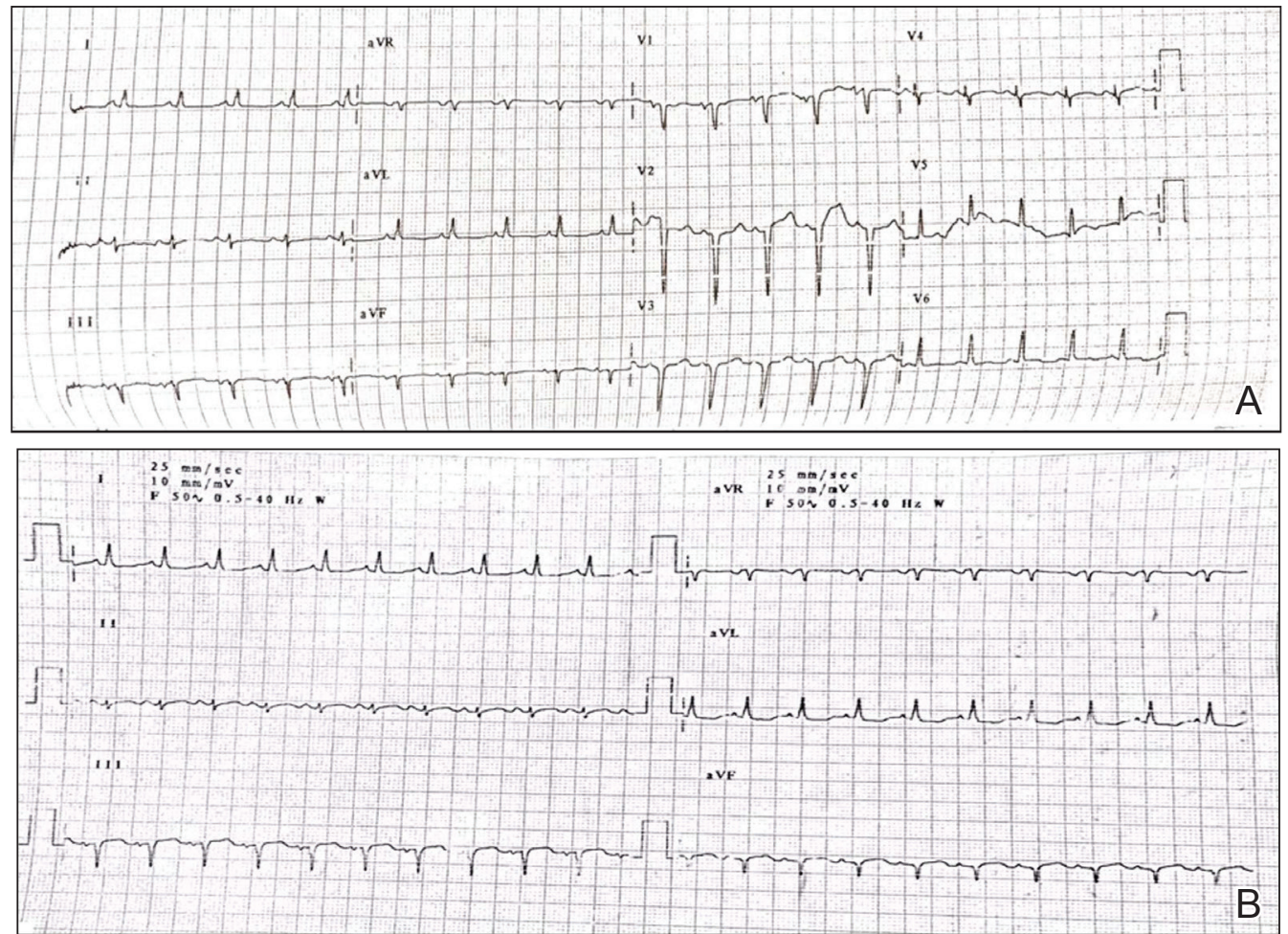

FIGURE 2. A, Baseline electrocardiogram. B, Electrocardiogram at the time of resuscitation.

including multiple attempts at cardioversion with up to $200 \mathrm{~J}$ (biphasic) and intravenous amiodarone infusion failed to revive the patient, and he died.

Proinflammatory cytokines such as IL-6 and tumor necrosis factor $\alpha$ are increased in young people with ventricular arrhythmias who have no evidence of myocardial injury (MI), suggesting an inflammatory background is involved. ${ }^{1}$ Psoriasis, a common immune-mediated inflammatory disease, has a chronic state of systemic inflammation with notably higher serum levels of tumor necrosis factor $\alpha$, IFN- $\gamma$, IL-6, IL-8, IL-12, and IL-18 compared to controls. ${ }^{2}$ This inflammation is not confined to skin but can involve blood vessels, joints, and the liver, as demonstrated by increased fluorodeoxyglucose uptake. ${ }^{3}$ It also seems to exert its influence on supraventricular beat development in patients with psoriasis who do not have a history of CVEs. ${ }^{4}$ Tumor necrosis factor $\alpha$ is one of the major cytokines playing a role in the inflammatory process of psoriasis. Studies have shown serum levels of tumor necrosis factor $\alpha$ to correlate with the clinical symptoms of 
heart failure and to supraventricular arrhythmia in animal models. ${ }^{4}$ Various extreme CVEs can be an expression of this ongoing dynamic process. It would be interesting to know which specific factors among these inflammatory cytokines lead to rhythm irregularities.

Another theory is that young patients may experience micro-MI during the disease course. These small infarcted areas may act as aberrant pulse generators or lead to conduction disturbances. One study found increased correct QT interval dispersion, a predictor of ventricular arrhythmias, to be associated with psoriasis. ${ }^{5}$ A nationwide population-based matched cohort study by Chiu et $\mathrm{al}^{6}$ revealed that patients with psoriasis have a higher risk for arrhythmia independent of traditional cardiovascular risk factors. Our patient also had severe unstable psoriasis for 8 years that may have led to increased accumulation of proarrhythmogenic cytokines in the heart and could have led to VT.

Acitretin as a potential cause of sudden cardiac death remains a possibility in our case; however, the exact mechanism leading to such sudden arrhythmia is lacking. Acitretin is known to increase serum triglycerides and cholesterol, specifically by shifting high-density lipoproteins to low-density lipoproteins, thereby increasing the risk for CVE. However, it takes time for such derangement to occur, eventually leading to CVE. Mittal et $\mathrm{al}^{7}$ reported a psoriasis patient who died secondary to MI after 5 days of low-dose acitretin. Lack of evidence makes acitretin a less likely cause of mortality.
We present a case of sudden cardiac death secondary to VT in a young patient with psoriasis and no other traditional cardiovascular risk factors. This case highlights the importance of being vigilant for adverse CVEs such as arrhythmia in psoriatic patients, especially in younger patients with severe unstable disease.

\section{REFERENCES}

1. Kowalewski M, Urban M, Mroczko B, et al. Proinflammatory cytokines (IL-6, TNF-alpha) and cardiac troponin I (cTnI) in serum of young people with ventricular arrhythmias. Pol Arch Med Wewn. 2002;108:647-651.

2. Arican O, Aral M, Sasmaz S, et al. Serum levels of TNF-alpha, IFN-gamma, IL-6, IL-8, IL-12, IL-17, and IL-18 in patients with active psoriasis and correlation with disease severity. Mediators Inflamm. 2005;2005:273-279.

3. Mehta NN, Yu Y, Saboury B, et al. Systemic and vascular inflammation in patients with moderate to severe psoriasis as measured by [18F]-fluorodeoxyglucose positron emission tomography -computed tomography (FDG-PET/CT): a pilot study. Arch Dermatol. 2011;147:1031-1039.

4. Markuszeski L, Bissinger A, Janusz I, et al. Heart rate and arrhythmia in patients with psoriasis vulgaris. Arch Med Res. 2007;38:64-69.

5. Simsek H, Sahin M, Akyol A, et al. Increased risk of atrial and ventricular arrhythmia in long-lasting psoriasis patients. ScientificWorldJournal. 2013;2013:901215.

6. Chiu HY, Chang WL, Huang WF, et al. Increased risk of arrhythmia in patients with psoriatic disease: a nationwide population-based matched cohort study. J Am Acad Dermatol. 2015;73:429-438.

7. Mittal R, Malhotra S, Pandhi P, et al. Efficacy and safety of combination acitretin and pioglitazone therapy in patients with moderate to severe chronic plaque-type psoriasis: a randomized, double-blind, placebocontrolled clinical trial. Arch Dermatol. 2009;145:387-393. 